\title{
Numerical Simulations of Water Quality Measurement Model in an Opened-Closed Reservoir with Contaminant Removal Mechanism
}

\author{
Kaboon Thongtha ${ }^{1,2}$ and Jaipong Kasemsuwan ${ }^{1,2}$ \\ ${ }^{1}$ Department of Mathematics, Faculty of Science, King Mongkut's Institute of Technology Ladkrabang, Bangkok 10520, Thailand \\ ${ }^{2}$ Centre of Excellence in Mathematics Commission on Higher Education (CHE), Si Ayutthaya Road, Bangkok 10400, Thailand
}

Correspondence should be addressed to Kaboon Thongtha; kaboon.t@gmail.com

Received 2 January 2018; Revised 7 March 2018; Accepted 13 March 2018; Published 2 May 2018

Academic Editor: Julian Poulter

Copyright (c) 2018 Kaboon Thongtha and Jaipong Kasemsuwan. This is an open access article distributed under the Creative Commons Attribution License, which permits unrestricted use, distribution, and reproduction in any medium, provided the original work is properly cited.

\begin{abstract}
The mathematical simulation of water contaminant measurement is often used to assess the water quality. The monitoring point placement for water quality measurement in an opened-closed reservoir can give accurate or inaccurate assessment. In this research, the mathematical model of the approximated water quality in an opened-closed reservoir with removal mechanism system is proposed. The water quality model consists of the hydrodynamic model and the dispersion model. The hydrodynamic model is used to describe the water current in the opened-closed reservoir. The transient advection-diffusion equation with removal mechanism provides the water pollutant concentration. The water velocity from the hydrodynamic model is plugged into the dispersion model. The finite difference techniques are used to approximate the solution of the water quality model. The proposed numerical simulations give a suitable area of zonal removal mechanism placement. The proposed simulations also give the overall and specified approximated water quality for each point and time when the exit gate is opened on the different periods of time. In addition, the proposed techniques can give a suitable period of time to open the exit gate to achieve a good agreement water quality by using contaminant removal mechanism.
\end{abstract}

\section{Introduction}

Field measurement and mathematical simulation are methods to detect the amount of the level of pollutants in water area. In water quality modeling for reservoir, the general governing equations used are the hydrodynamic model and the dispersion model. The two-dimensional shallow water equation and the advection-diffusion-reaction equation govern the first and the second models, respectively.

The several numerical techniques for solving such models were available. In [1-3], they used the hydrodynamic model and the dispersion model with the finite element method to approximate the velocity of the water current in bay, estuaries, and open reservoir, respectively. In [4], the finite element method was used for solving the water pollution levels to the optimal control of the water treatment plants to achieve minimum cost. In [5], the method of the characteristic technique combined with Galerkin finite element method is used to solve the shallow water mass transport problems. In $[6,7]$, the numerical techniques are used to solve the nonuniform flow of stream water quality model with the advection-dispersionreaction equations. In [8], the Crank-Nicolson method is used to solve the hydrodynamic model and the backward time central space (BTCS) for the dispersion model. In [9], the approximated solutions of the hydrodynamic model and advection-diffusion-reaction equation in a uniform reservoir are proposed. In [10], a nondimensional form of the hydrodynamic model with variable coefficients using Lax-Wendroff method is presented. In [11], the Lax-Wendroff finite difference method is also proposed to approximate the water elevation and water flow velocity with a rectangular domain. In [12], the mathematical models and numerical methods for approximating water current and pollutant concentration level in Rama-nine reservoirs are presented. In [13], 
the Lax-Wendroff method for solving the dimensional form of shallow water equation in spherical model with Matlab program is proposed. In [14], an analytical solution to a hydrodynamic model in an open uniform reservoir with the specified tidal wave functions is proposed.

In this research, the mathematical models for water quality measurement which consist of the hydrodynamic model and the dispersion model, used to simulate water quality in a water flow systems, were considered. The first is a hydrodynamic model that provides the water current and the elevation of water in an opened-closed reservoir. The second is a dispersion model that gives the concentration of pollutant in an opened-closed reservoir with the contaminant removal mechanism. For numerical techniques, we used the Lax-Wendroff method to the system of the hydrodynamic model and the forward in time central in space (FTCS) to the dispersion model. The results from the shallow water equation of the hydrodynamic model are the water flow velocity which are input data for advection-diffusionreaction equation which provides the level of pollutant concentration field. Averaging the equation over the depth with anisotropic bottom topography and discarding the term due to the Coriolis force, surface wind effect, and external forces, it follows that the two-dimensional shallow water and advection-diffusion-reaction equations are applicable.

\section{Water Quality Measurement in Opened-Closed Reservoir with Removal Mechanism Model}

The mathematical models for water quality measurement in opened-closed reservoir with removal mechanism are described. They are used to simulate time-varying pollutant levels caused by waste water discharges from external source into an opened-closed reservoir with removal mechanism and drain water at the exit gate. The first model is a hydrodynamic model that determined the velocity and elevation of the water at any locations in the reservoir with anisotropic bottom topography, while the second model is a pollutant dispersion with removal mechanism model that determined the pollutant level at any points in the reservoir.

2.1. Hydrodynamic Model. The two-dimensional unsteady water current into and out of the reservoir can be determined by using the system of shallow water equations as the conservation of mass and conservation of momentum. It is taken into account that the equations of the system of shallow water can be derived from depth-averaging NavierStokes equations in the vertical direction, neglecting the diffusion of momentum due to turbulence and discarding the terms expressing the effects of friction, surface wind, Coriolis factor, and shearing stresses. The continuity and momentum equation governs the hydrodynamic behavior of the reservoir [15]. The well known two-dimensional shallow water equations are $[1,2,13,16]$

$$
\frac{\partial h}{\partial t}+\frac{\partial}{\partial x}(u h)+\frac{\partial}{\partial y}(v h)=0
$$

$$
\begin{aligned}
& \frac{\partial(u h)}{\partial t}+\frac{\partial\left(u^{2} h+(1 / 2) g h^{2}\right)}{\partial x}+\frac{\partial(u v h)}{\partial y}=0 \\
& \frac{\partial(v h)}{\partial t}+\frac{\partial(u v h)}{\partial x}+\frac{\partial\left(v^{2} h+(1 / 2) g h^{2}\right)}{\partial y}=0
\end{aligned}
$$

where $x$ is the longitudinal distance along the reservoir $(\mathrm{m})$, $y$ is the transverse distance along the reservoir $(\mathrm{m}), t$ is the time (s), $\eta(x, y)$ is the depth measured from the mean water level to the reservoir bed $(\mathrm{m}), \zeta(x, y, t)$ is the elevation of water surface from the mean water level in reservoir $(\mathrm{m})$, $h(x, y, t)=\eta(x, y)+\zeta(x, y, t)$ is the elevation of water surface measured from the mean water level to the bed of the reservoir, $u(x, y, t)$ is velocity in $x$-direction $(\mathrm{m} / \mathrm{s}), v(x, y, t)$ is velocity in $y$-direction $(\mathrm{m} / \mathrm{s})$, and $g$ is gravitational constant $\left(9.81 \mathrm{~m} / \mathrm{s}^{2}\right)$, for all $(x, y, t) \in \Omega=[0, l] \times[0, m] \times[0, T]$.

Such independent variables $x, y$, and $t$ make up the special dimensions and time. The dependent variables are the depth $h$ with respect to the surface and the two-dimensional velocities $u$ and $v$. The partial derivatives taken with respect to the same term $(\partial t, \partial x, \partial y)$ are grouped into vectors and rewritten as a single hyperbolic partial differential $[13,15]$,

$$
\frac{\partial H}{\partial t}+\frac{\partial U}{\partial x}+\frac{\partial V}{\partial y}=0
$$

where

$$
\begin{aligned}
& H=\left(\begin{array}{l}
h \\
u h \\
v h
\end{array}\right), \\
& U=\left(\begin{array}{c}
u h \\
u^{2} h+\frac{1}{2} g h^{2} \\
u v h
\end{array}\right), \\
& V=\left(\begin{array}{c}
v h \\
u v h \\
v^{2} h+\frac{1}{2} g h^{2}
\end{array}\right) .
\end{aligned}
$$

\subsection{Dispersion Model}

2.2.1. Water Pollutant Dispersion Model. Applying the distributed pollutant process, including the transportation and diffusion, we have the mass transfer equation. There is a representation simplified by averaging the equation over the depth, generating the advection-diffusion equation as follows [4]:

$$
\frac{\partial C}{\partial t}+u \frac{\partial C}{\partial x}+v \frac{\partial C}{\partial y}=D\left(\frac{\partial^{2} C}{\partial^{2} x}+\frac{\partial^{2} C}{\partial^{2} y}\right),
$$

where $C(x, y, t)$ is the depth averaged water pollutant concentration at the point $(x, y)$ and at the time $t\left(\mathrm{~kg} / \mathrm{m}^{3}\right)$ and $D$ is the pollutant dispersion coefficient $\left(\mathrm{m} / \mathrm{s}^{2}\right)$. 


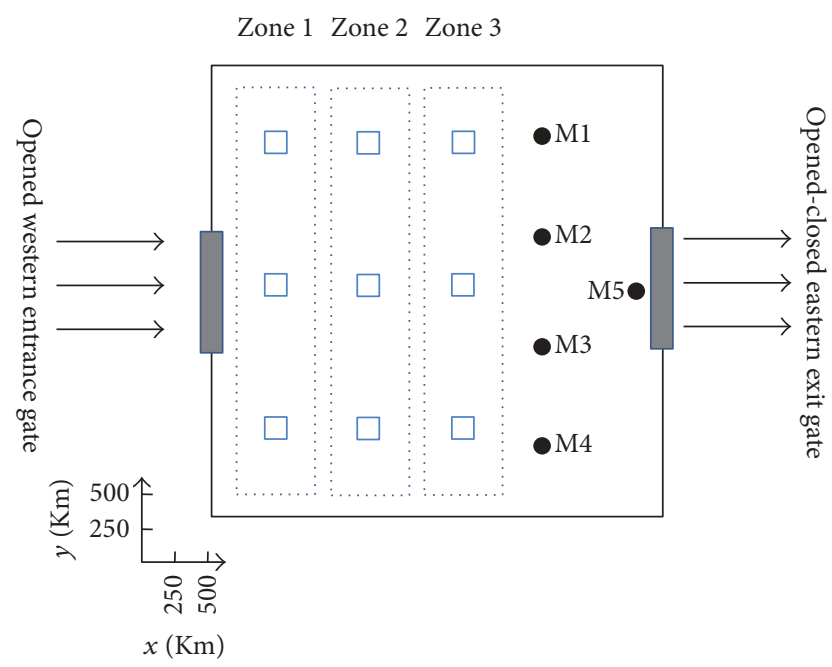

FIGURE 1: An opened-closed reservoir with contaminant removal mechanism zones and monitoring points M1, M2, M3, M4, and M5.

2.2.2. Water Pollutant Dispersion with Removal Mechanism Model. The mechanisms of pollutant removal are introduced by decaying chemical reaction and absorptive reduction. A representation is modified by generating the advectiondiffusion-reaction with sink term [2],

$$
\begin{aligned}
\frac{\partial C}{\partial t}+u \frac{\partial C}{\partial x}+v \frac{\partial C}{\partial y}= & D\left(\frac{\partial^{2} C}{\partial^{2} x}+\frac{\partial^{2} C}{\partial^{2} y}\right)-R C \\
& -Q(x, y, t)
\end{aligned}
$$

where $C(x, y, t)$ is the depth averaged water pollutant concentration at the point $(x, y)$ and at the time $t\left(\mathrm{~kg} / \mathrm{m}^{3}\right), D$ is the pollutant dispersion coefficient $\left(\mathrm{m} / \mathrm{s}^{2}\right), R \geq 0$ is the water pollutant decaying rate $\left(\mathrm{s}^{-1}\right)$, and $Q(x, y, t)$ is the decreasing rate of water pollutant concentration due to a water pollutant $\operatorname{sink}\left(\mathrm{kg} / \mathrm{m}^{3} \mathrm{~s}\right)$.

2.3. Initial and Boundary Conditions of Hydrodynamic Model in Opened-Closed Reservoir. The initial conditions of (2) are assumed to be motionless,

$$
\begin{gathered}
u(x, y, 0)=0, \\
v(x, y, 0)=0, \\
h(x, y, 0)=0 .
\end{gathered}
$$

An opened-closed reservoir supplied by the outer water wave is going to flow into the reservoir such that the water is drained as shown in Figure 1. The elevation of water on the opened gate is assumed to be a wave maker function, say $f$. The elevation of water on the exit gates is assumed to be $\partial h / \partial x=\kappa$, where $\kappa<0$ is the rate of change between inner and outer water level through the eastern exit gate.

The boundary conditions of the model in an openedclosed reservoir are assumed as follows:

(i) $u=0, \partial v / \partial y=0$, and $\partial h / \partial y=0$, for all $(x, y)$ lie on the horizontal edges of the reservoir. (ii) $v=0, \partial u / \partial x=0$, and $\partial h / \partial x=0$ for all $(x, y)$ lie on the vertical edges of the reservoir.

(iii) $v=0, \partial u / \partial x=0$, and $h=f$ for all $(x, y)$ lie on the flowing water into the opened entrance gate.

(iv) $v=0, \partial u / \partial x=u_{e}$, and $\partial h / \partial x=\kappa$ for all $(x, y)$ lie on the flowing water at the opened-closed exit gate. The initial and boundary conditions are also shown in Figure 2.

2.4. Initial and Boundary Conditions of Water Pollutant Dispersion Model in Opened-Closed Reservoir. The initial pollutant concentration in reservoir is $c_{0}\left(\mathrm{~kg} / \mathrm{m}^{3}\right)$. The water pollutant is discharged from the open gate into the openedclosed reservoir which are assumed to be $C(x, y, t)=$ $c_{1}$, where $c_{1}\left(\mathrm{~kg} / \mathrm{m}^{3}\right)$ is the averaged discharged pollutant concentration along the entrance gate. The opened-closed reservoir drain water at the exit gate by assuming rate of water drain as $\partial C / \partial x=-c_{2}$, where $c_{2}$ is a nonnegative rate of change of pollutant concentration across the exit gate. The embankment of reservoir is a nonabsorbing boundary bank. Consequently, there is no rate of change of pollutant concentration at the boundary of opened-closed reservoir, $\partial C / \partial n=0$, where $n$ is a normal vector, as shown in Figure 3 .

\subsection{Removal Pollutant Concentration Mechanism in Water} Pollutant Dispersion Model. The removal terms can model a variety of different phenomena, that is, the removal of pollutant concentration in (5) with absorptive rate function $Q=Q(x, y, t)\left(\mathrm{kg} / \mathrm{m}^{3} \mathrm{~s}\right)$, where $Q$ is a nonnegative real value function.

The term $R$ in (5) arises when there is pollutant concentration loss from a water surface; the reaction term is used to describe the decaying rate of pollutant concentration, where $R$ is a nonnegative constant.

\section{Numerical Techniques}

The hydrodynamic model provides the velocity field and elevation of the water. Then the calculated results will be input into the dispersion model that provides the pollutant concentration results.

3.1. Lax-Wendroff Method for the Hydrodynamic Model. We apply the numerical method for solving the single hyperbolic partial differential equations known as the Lax-Wendroff method. The Lax-Wendroff method involves starting to calculate a first half step and then using the result from the half step to calculate the full step $[7,8]$. To find the water velocity and water elevation, we now discretize (2) on a rectangle reservoir with length $l$ and width $m$. The domain $[0, l] \times[0, m]$ is divided into $L$ and $M$ subintervals such that $L \Delta x=l$ and $M \Delta y=m$, respectively. The time intervals $[0, k]$ are also divided into $T$ subintervals such that $T \Delta t=k$. We can then approximate $H\left(x_{i}, y_{j}, t_{n}\right)$ by $H_{i, j}^{n}$ and the value of the difference approximation of $H(x, y, t)$ at points $x=i \Delta x$ and $y=j \Delta y$ and time $t=n \Delta t$ where $0 \leq i \leq L, 0 \leq j \leq M$, and $0 \leq n \leq T$, similarly defined for $U_{i, j}^{n}$ and $V_{i, j}^{n}$. The grid 


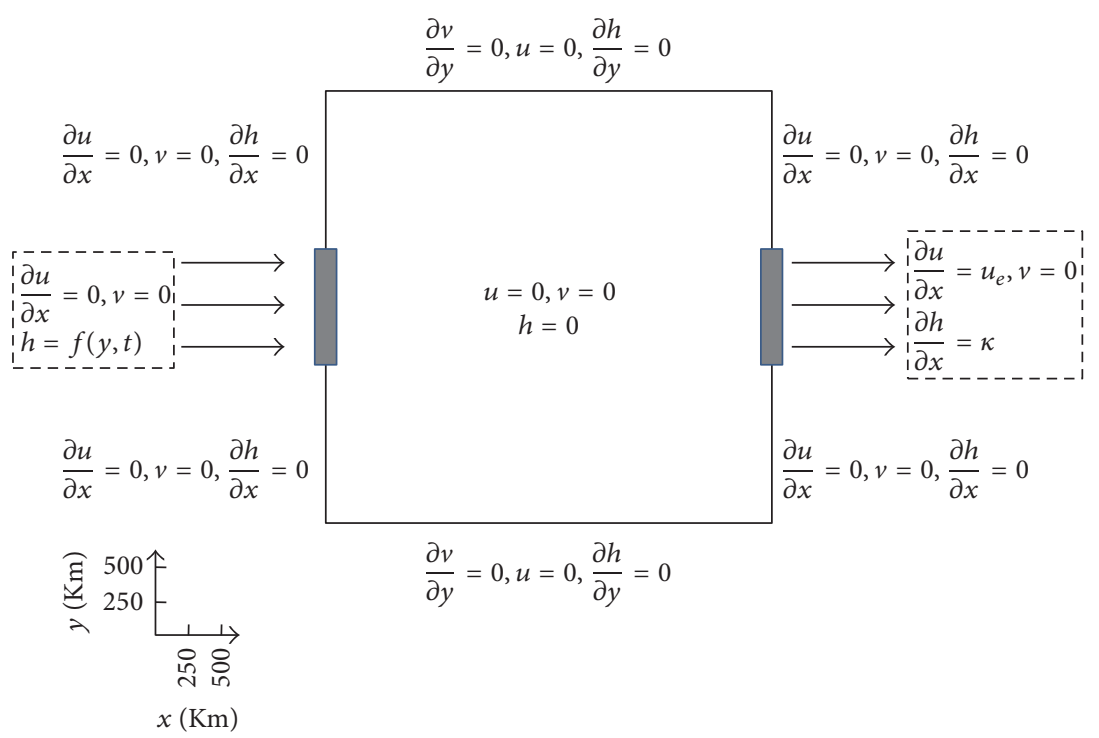

FIGURE 2: Initial and boundary conditions of hydrodynamic model in opened-closed reservoir.

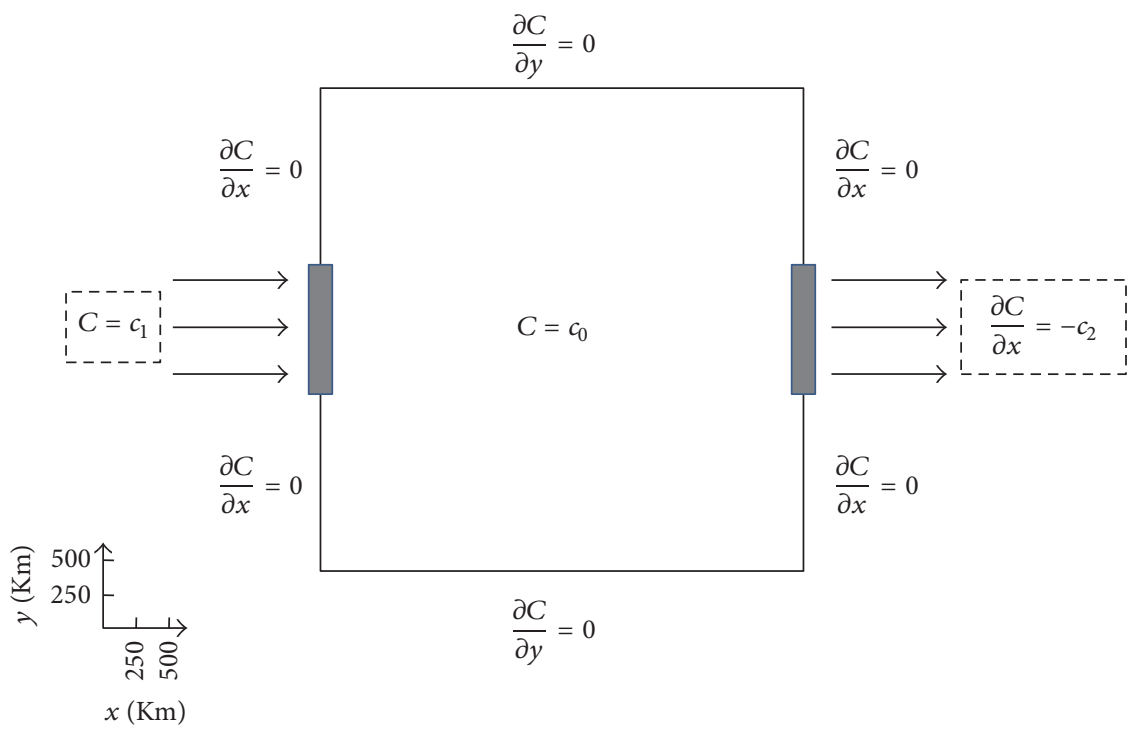

FIGURE 3: The initial and boundary conditions of water pollutant dispersion model in opened-closed reservoir.

points are defined by $x_{i}=i \Delta x$ for all $i=0,1,2, \ldots, L$, $y_{j}=j \Delta y$ for all $j=0,1,2, \ldots, M$, and $t_{n}=n \Delta t$ for all $n=0,1,2, \ldots, T$ in which $L, M$, and $T$ are positive integers. Using the Lax-Wendroff method $[7,8]$ on $(2)$, we can obtain the following finite difference equation of hyperbolic partial differential equations.

The first half step defines values of $H$ at time step $n+1 / 2$ and the midpoint of the grid cell; the first half steps are stored in separate matrices $H_{x}$ and $H_{y}$ to be reintroduced during the second half step as shown in Figure 4. The first half steps for $U$ and $V$ are approximated in a similar process and are stored in $U_{x}, U_{y}$ and $V_{x}, V_{y}$ as follows $[13,15]$ :

$$
\begin{aligned}
& H_{x_{i+1 / 2, j}}^{n+1 / 2}=\frac{1}{2}\left(H_{i+1, j}^{n}+H_{i, j}^{n}\right)-\frac{\Delta t}{2 \Delta x}\left(U_{i+1, j}^{n}-U_{i, j}^{n}\right), \\
& H_{y_{i, j+1 / 2}}^{n+1 / 2}=\frac{1}{2}\left(H_{i, j+1}^{n}+H_{i, j}^{n}\right)-\frac{\Delta t}{2 \Delta y}\left(V_{i, j+1}^{n}-V_{i, j}^{n}\right),
\end{aligned}
$$

$$
\begin{aligned}
& U_{x_{i+1 / 2, j}^{n+1 / 2}}^{n+}=\frac{1}{2}\left(U_{i+1, j}^{n}+U_{i, j}^{n}\right) \\
& \quad-\frac{\Delta t}{2 \Delta x}\left[\left(\left(U_{i+1, j}^{n}\right)^{2} H_{i+1, j}^{n}-\left(U_{i, j}^{n}\right)^{2} H_{i, j}^{n}\right)\right. \\
& \left.\quad+\left(\frac{g}{2}\left(H_{i+1, j}^{n}\right)^{2}-\frac{g}{2}\left(H_{i, j}^{n}\right)^{2}\right)\right], \\
& U_{y_{i, j+1 / 2}^{n+1 / 2}}^{n}=\frac{1}{2}\left(U_{i, j+1}^{n}+U_{i, j}^{n}\right)-\frac{\Delta t}{2 \Delta y}\left[\left(U_{i, j+1}^{n} V_{i, j+1}^{n} H_{i, j+1}^{n}\right)\right. \\
& \left.\quad-\left(U_{i, j}^{n} V_{i, j}^{n} H_{i, j}^{n}\right)\right], \\
& V_{x_{i+1 / 2, j}}^{n+1 / 2}=\frac{1}{2}\left(V_{i+1, j}^{n}+V_{i, j}^{n}\right)-\frac{\Delta t}{2 \Delta x}\left[\left(U_{i+1, j}^{n} V_{i+1, j}^{n} H_{i+1, j}^{n}\right)\right. \\
& \left.\quad-\left(U_{i, j}^{n} V_{i, j}^{n} H_{i, j}^{n}\right)\right],
\end{aligned}
$$




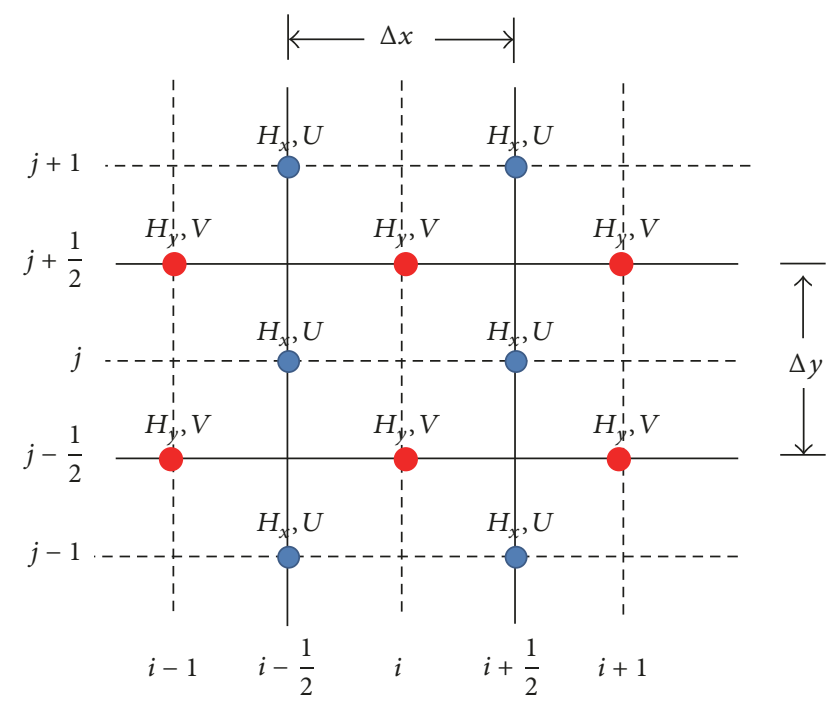

Figure 4: The values of vector $H_{x}, H_{y}, U$, and $V$ represented the solution at the midpoints of the grid cell.

$$
\begin{aligned}
& V_{y_{i, j+1 / 2}^{n+1 / 2}}=\frac{1}{2}\left(V_{i, j+1}^{n}+V_{i, j}^{n}\right) \\
& \quad-\frac{\Delta t}{2 \Delta y}\left[\left(\left(U_{i, j+1}^{n}\right)^{2} H_{i, j+1}^{n}-\left(U_{i, j}^{n}\right)^{2} H_{i, j}^{n}\right)\right. \\
& \left.\quad+\left(\frac{g}{2}\left(H_{i, j+1}^{n}\right)^{2}-\frac{g}{2}\left(H_{i, j}^{n}\right)^{2}\right)\right] .
\end{aligned}
$$

The second half step completes the time step $n+1$ by using the values calculated in the first half step to calculate new values at the centre of the grid cell $[13,15]$ :

$$
\begin{aligned}
H_{i, j}^{n+1} & =H_{i, j}^{n}-\frac{\Delta t}{\Delta x}\left(U_{x_{i+1 / 2, j}}^{n+1 / 2}-U_{x_{i-1 / 2, j}}^{n+1 / 2}\right)-\frac{\Delta t}{\Delta y}\left(V_{y_{i, j+1 / 2}}^{n+1 / 2}\right. \\
- & \left.V_{y_{i, j-1 / 2}}^{n+1 / 2}\right), \\
U_{i, j}^{n+1} & =U_{i, j}^{n} \\
- & \frac{\Delta t}{\Delta x}\left[\left(\left(U_{x_{i+1 / 2, j}}^{n+1 / 2}\right)^{2}\left(H_{x_{i+1 / 2, j}}^{n+1 / 2}\right)+\frac{g}{2}\left(H_{x_{i+1 / 2, j}}^{n+1 / 2}\right)^{2}\right)\right. \\
- & \left.\left(\left(U_{x_{i-1 / 2, j}}^{n+1 / 2}\right)^{2}\left(H_{x_{i-1 / 2, j}}^{n+1 / 2}\right)+\frac{g}{2}\left(H_{x_{i-1 / 2, j}}^{n+1 / 2}\right)^{2}\right)\right] \\
- & \frac{\Delta t}{\Delta y}\left[\left(U_{y_{i, j+1 / 2}}^{n+1 / 2}\right)\left(V_{y_{i, j}+1 / 2}^{n+1 / 2}\right)\left(H_{y_{i, j+1 / 2}}^{n+1 / 2}\right)\right. \\
- & \left.\left(U_{y_{i, j-1 / 2}}^{n+1 / 2}\right)\left(V_{y_{i, j-1 / 2}}^{n+1 / 2}\right)\left(H_{y_{i, j-1 / 2}}^{n+1 / 2}\right)\right], \\
V_{i, j}^{n+1} & =V_{i, j}^{n}-\frac{\Delta t}{\Delta x}\left[\left(U_{x_{i+1 / 2, j}}^{n+1 / 2}\right)\left(V_{x_{i+1 / 2, j}}^{n+1 / 2}\right)\left(H_{x_{i+1 / 2, j}}^{n+1 / 2}\right)\right. \\
- & \left.\left(U_{x_{i-1 / 2, j}}^{n+1 / 2}\right)\left(V_{x_{i-1 / 2, j}}^{n+1 / 2}\right)\left(H_{x_{i-1 / 2, j}}^{n+1 / 2}\right)\right] \\
- & \frac{\Delta t}{\Delta y}\left[\left(\left(V_{y_{i, j+1 / 2}}^{n+1 / 2}\right)^{2}\left(H_{y_{i, j+1 / 2}}^{n+1 / 2}\right)+\frac{g}{2}\left(H_{y_{i, j+1 / 2}}^{n+1 / 2}\right)^{2}\right)\right. \\
& \left.\left.\left(H_{y_{i, j-1 / 2}}^{n+1 / 2}\right)+\frac{g}{2}\left(H_{y_{i, j-1 / 2}}^{n+1 / 2}\right)^{2}\right)\right] .
\end{aligned}
$$

The finite difference method computes a numerical approximation to the boundary conditions of the reservoir. For the left boundary condition, we have $i=0$ and $1 \leq j \leq M$, letting $H_{0, j}^{n}=H_{1, j}^{n}, U_{0, j}^{n}=U_{1, j}^{n}$, and $V_{0, j}^{n}=V_{1, j}^{n}$. Substituting the approximate unknown vector nodes $H_{0, j}^{n}, U_{0, j}^{n}$, and $V_{0, j}^{n}$ of the left boundary into (7), (9), and (11), respectively,

$$
\begin{aligned}
& H_{x_{1 / 2, j}}^{n+1 / 2}=\frac{1}{2}\left(H_{1, j}^{n}+H_{0, j}^{n}\right)-\frac{\Delta t}{2 \Delta x}\left(U_{1, j}^{n}-U_{0, j}^{n}\right)=H_{1, j}^{n}, \\
& U_{x_{1 / 2, j}}^{n+1 / 2}=\frac{1}{2}\left(U_{1, j}^{n}+U_{0, j}^{n}\right) \\
& \quad-\frac{\Delta t}{2 \Delta x}\left[\left(\left(U_{1, j}^{n}\right)^{2} H_{1, j}^{n}-\left(U_{0, j}^{n}\right)^{2} H_{0, j}^{n}\right)\right. \\
& \left.+\left(\frac{g}{2}\left(H_{1, j}^{n}\right)^{2}-\frac{g}{2}\left(H_{0, j}^{n}\right)^{2}\right)\right]=U_{1, j}^{n}, \\
& V_{x_{1 / 2, j}}^{n+1 / 2}=\frac{1}{2}\left(V_{1, j}^{n}+V_{0, j}^{n}\right)-\frac{\Delta t}{2 \Delta x}\left[\left(U_{1, j}^{n} V_{1, j}^{n} H_{1, j}^{n}\right)\right. \\
& \left.\quad-\left(U_{0, j}^{n} V_{0, j}^{n} H_{0, j}^{n}\right)\right]=V_{1, j}^{n} .
\end{aligned}
$$

For the right boundary condition, we have $i=L$ and $1 \leq j \leq$ $M$, letting $H_{L+1, j}^{n}=H_{L, j}^{n}, U_{L+1, j}^{n}=U_{L, j}^{n}$, and $V_{L+1, j}^{n}=V_{L, j}^{n}$ Substituting the approximate unknown vector nodes $H_{L+1, j}^{n}$, $U_{L+1, j}^{n}$, and $V_{L+1, j}^{n}$ of the right boundary into (7), (9), and (11), respectively,

$$
\begin{aligned}
& H_{x_{L+1 / 2, j}}^{n+1 / 2}=\frac{1}{2}\left(H_{L+1, j}^{n}+H_{L, j}^{n}\right)-\frac{\Delta t}{2 \Delta x}\left(U_{L+1, j}^{n}-U_{L, j}^{n}\right) \\
& \quad=H_{L, j}^{n}, \\
& U_{x_{L+1 / 2, j}}^{n+1 / 2}=\frac{1}{2}\left(U_{L+1, j}^{n}+U_{L, j}^{n}\right) \\
& \quad-\frac{\Delta t}{2 \Delta x}\left[\left(\left(U_{L+1, j}^{n}\right)^{2} H_{L+1, j}^{n}-\left(U_{L, j}^{n}\right)^{2} H_{L, j}^{n}\right)\right. \\
& \left.+\left(\frac{g}{2}\left(H_{L+1, j}^{n}\right)^{2}-\frac{g}{2}\left(H_{L, j}^{n}\right)^{2}\right)\right]=U_{L, j}^{n}, \\
& V_{x_{L+1 / 2, j}}^{n+1 / 2}=\frac{1}{2}\left(V_{L+1, j}^{n}+V_{L, j}^{n}\right) \\
& \quad-\frac{\Delta t}{2 \Delta x}\left[\left(U_{L+1, j}^{n} V_{L+1, j}^{n} H_{L+1, j}^{n}\right)-\left(U_{L, j}^{n} V_{L, j}^{n} H_{L, j}^{n}\right)\right] \\
& =V_{L, j}^{n} .
\end{aligned}
$$

For the lower boundary condition, we have $1 \leq i \leq L$ and $j=$ 0 , letting $H_{i, 0}^{n}=H_{i, 1}^{n}, U_{i, 0}^{n}=U_{i, 1}^{n}$, and $V_{i, 0}^{n}=V_{i, 1}^{n}$ Substituting the approximate unknown vector nodes $H_{i, 0}^{n}, U_{i, 0}^{n}$, and $V_{i, 0}^{n}$ of the lower boundary into (8), (10), and (12), respectively,

$$
\begin{aligned}
& H_{y_{i, 1 / 2}}^{n+1 / 2}=\frac{1}{2}\left(H_{i, 1}^{n}+H_{i, 0}^{n}\right)-\frac{\Delta t}{2 \Delta y}\left(V_{i, 1}^{n}-V_{i, 0}^{n}\right)=H_{i, 1}^{n}, \\
& U_{y_{i, 1 / 2}^{n+1 / 2}}^{n+}=\frac{1}{2}\left(U_{i, 1}^{n}+U_{i, 0}^{n}\right)-\frac{\Delta t}{2 \Delta y}\left[\left(U_{i, 1}^{n} V_{i, 1}^{n} H_{i, 1}^{n}\right)\right. \\
& \left.\quad-\left(U_{i, 0}^{n} V_{i, 0}^{n} H_{i, 0}^{n}\right)\right]=U_{i, 1}^{n},
\end{aligned}
$$




$$
\begin{aligned}
& V_{y_{i, 1 / 2}}^{n+1 / 2}=\frac{1}{2}\left(V_{i, 1}^{n}+V_{i, 0}^{n}\right) \\
& -\frac{\Delta t}{2 \Delta y}\left[\left(\left(U_{i, 1}^{n}\right)^{2} H_{i, 1}^{n}-\left(U_{i, 0}^{n}\right)^{2} H_{i, 0}^{n}\right)\right. \\
& \left.+\left(\frac{g}{2}\left(H_{i, 1}^{n}\right)^{2}-\frac{g}{2}\left(H_{i, 0}^{n}\right)^{2}\right)\right]=V_{i, 1}^{n} .
\end{aligned}
$$

For the upper boundary condition, we have $1 \leq i \leq L$ and $j=$ $M$, letting $H_{i, M+1}^{n}=H_{i, M}^{n}, U_{i, M+1}^{n}=U_{i, M}^{n}$, and $V_{i, M+1}^{n}=V_{i, M}^{n}$. Substituting the approximate unknown vector nodes $H_{i, M+1}^{n}$, $U_{i, M+1}^{n}$, and $V_{i, M+1}^{n}$ of the upper boundary into (8), (10), and (12), respectively,

$$
\begin{aligned}
& H_{y_{i, M+1 / 2}}^{n+1 / 2}=\frac{1}{2}\left(H_{i, M+1}^{n}+H_{i, M}^{n}\right)-\frac{\Delta t}{2 \Delta y}\left(V_{i, M+1}^{n}-V_{i, M}^{n}\right) \\
& \quad=H_{i, M}^{n}, \\
& U_{y_{i, M+1 / 2}}^{n+1 / 2}=\frac{1}{2}\left(U_{i, M+1}^{n}+U_{i, M}^{n}\right) \\
& \quad-\frac{\Delta t}{2 \Delta y}\left[\left(U_{i, M+1}^{n} V_{i, M+1}^{n} H_{i, M+1}^{n}\right)-\left(U_{i, M}^{n} V_{i, M}^{n} H_{i, M}^{n}\right)\right] \\
& \quad=U_{i, M}^{n}, \\
& V_{y_{i, M+1 / 2}}^{n+1 / 2}=\frac{1}{2}\left(V_{i, M+1}^{n}+V_{i, M}^{n}\right) \\
& \quad-\frac{\Delta t}{2 \Delta y}\left[\left(\left(U_{i, M+1}^{n}\right)^{2} H_{i, M+1}^{n}-\left(U_{i, M}^{n}\right)^{2} H_{i, M}^{n}\right)\right. \\
& \left.\quad+\left(\frac{g}{2}\left(H_{i, M+1}^{n}\right)^{2}-\frac{g}{2}\left(H_{i, M}^{n}\right)^{2}\right)\right]=V_{i, M}^{n} .
\end{aligned}
$$

3.2. Forward Time Central Space Method for Water Pollutant Dispersion with Removal Mechanism Model. We can then approximate $C\left(x_{i}, y_{j}, t_{n}\right)$ by $C_{i, j}^{n}$; the value of the difference approximation of $C(x, y, t)$ at point the grid point $\left(x_{i}, y_{j}, t_{n}\right)$ is defined by $x_{i}=i \Delta x$ for all $i=0,1,2, \ldots, L, y_{j}=$ $j \Delta y$ for all $j=0,1,2, \ldots, M$, and $t_{n}=n \Delta t$ for all $n=$ $0,1,2, \ldots, T$, in which $L, M$, and $T$ are positive integers. We use the forward differences in time and central difference in space in advection-diffusion equation; we get the finite difference equations as follows [15]:

$$
\begin{aligned}
& C \approx C_{i, j}^{n}, \\
& \frac{\partial C}{\partial t} \approx \frac{C_{i, j}^{n+1}-C_{i, j}^{n}}{\Delta t}, \\
& \frac{\partial C}{\partial x} \approx \frac{C_{i+1, j}^{n}-C_{i-1, j}^{n}}{2 \Delta x}, \\
& \frac{\partial C}{\partial y} \approx \frac{C_{i, j+1}^{n}-C_{i, j-1}^{n}}{2 \Delta y}, \\
& \frac{\partial^{2} C}{\partial x^{2}} \approx \frac{C_{i+1, j}^{n}-2 C_{i, j}^{n}+C_{i-1, j}^{n}}{(\Delta x)^{2}},
\end{aligned}
$$

$$
\begin{aligned}
\frac{\partial^{2} C}{\partial y^{2}} & \approx \frac{C_{i, j+1}^{n}-2 C_{i, j}^{n}+C_{i, j-1}^{n}}{(\Delta y)^{2}}, \\
u & \approx U_{i, j}^{n}, \\
v & \approx V_{i, j}^{n}, \\
Q & =Q_{i, j}^{n} .
\end{aligned}
$$

Taking the forward time central space technique [15] into (5), we get the following finite difference equation:

$$
\begin{aligned}
& \frac{C_{i, j}^{n+1}-C_{i, j}^{n}}{\Delta t}+u_{i, j}^{n}\left(\frac{C_{i+1, j}^{n}+C_{i-1, j}^{n}}{2 \Delta x}\right) \\
& +v_{i, j}^{n}\left(\frac{C_{i, j+1}^{n}+C_{i, j-1}^{n}}{2 \Delta y}\right) \\
& =D\left(\frac{C_{i+1, j}^{n}-2 C_{i, j}^{n}+C_{i-1, j}^{n}}{(\Delta x)^{2}}\right. \\
& \left.+\frac{C_{i, j+1}^{n}-2 C_{i, j}^{n}+C_{i, j-1}^{n}}{(\Delta y)^{2}}\right)-R C_{i, j}^{n}-Q_{i, j}^{n} .
\end{aligned}
$$

Rearrangement of (19) gives

$$
\begin{aligned}
C_{i, j}^{n}= & \left(\gamma_{x}-\lambda_{x} u_{i, j}^{n}\right) C_{i+1, j}^{n}+\left(\gamma_{y}-\lambda_{y} v_{i, j}^{n}\right) C_{i, j+1}^{n} \\
& +\left(\gamma_{x}+\lambda_{x} u_{i, j}^{n}\right) C_{i-1, j}^{n}+\left(\gamma_{y}+\lambda_{y} v_{i, j}^{n}\right) C_{i, j-1}^{n} \\
& +\left(1-2 \gamma_{x}-2 \gamma_{y}-R \Delta t\right) C_{i, j}^{n}-Q_{i, j}^{n} \Delta t
\end{aligned}
$$

where $\lambda_{x}=\Delta t / 2 \Delta x, \lambda_{y}=\Delta t / 2 \Delta y, \gamma_{x}=D \Delta t /(\Delta x)^{2}$, $\gamma_{y}=D \Delta t /(\Delta y)^{2}, D$ is the diffusion coefficient $\left(\mathrm{m}^{2} / \mathrm{s}\right), R$ is the substance decaying rate $\left(\mathrm{s}^{-1}\right)$, and $Q(x, y, t)$ is the decreasing rate of water pollutant concentration due to a water pollutant sink $\left(\mathrm{kg} / \mathrm{m}^{3} \mathrm{~s}\right)$.

If $C_{i, j}^{n}$ is on the boundary of the opened-closed reservoir, it is calculated by applying the backward difference scheme to the right boundary condition and the upper boundary condition. We also take the forward difference scheme to the left boundary condition and the lower boundary condition.

For the left boundary condition, we have $i=1$ and $1 \leq j \leq$ $M$, letting $C_{0, j}^{n}=C_{1, j}^{n}$. Substitute the approximate unknown vector nodes $C_{0, j}^{n}$ of the left boundary into (20) as follows:

$$
\begin{aligned}
& \frac{C_{1, j}^{n+1}-C_{1, j}^{n}}{\Delta t}+u_{1, j}^{n}\left(\frac{C_{2, j}^{n}+C_{0, j}^{n}}{2 \Delta x}\right) \\
& \quad+v_{1, j}^{n}\left(\frac{C_{1, j+1}^{n}+C_{1, j-1}^{n}}{2 \Delta y}\right) \\
& =D\left(\frac{C_{2, j}^{n}-C_{1, j}^{n}}{(\Delta x)^{2}}+\frac{C_{1, j+1}^{n}-2 C_{1, j}^{n}+C_{1, j-1}^{n}}{(\Delta y)^{2}}\right) \\
& \quad-R C_{1, j}^{n}-Q_{1, j}^{n} .
\end{aligned}
$$


For the right boundary condition, we have $i=L$ and $1 \leq j \leq$ $M$, letting $C_{L+1, j}^{n}=C_{L, j}^{n}$. Substitute the approximate unknown vector nodes $C_{L+1, j}^{n}$ of the right boundary into (20) as follows:

$$
\begin{aligned}
& \frac{C_{L, j}^{n+1}-C_{L, j}^{n}}{\Delta t}+u_{L, j}^{n}\left(\frac{C_{L+1, j}^{n}+C_{L-1, j}^{n}}{2 \Delta x}\right) \\
& \quad+v_{L, j}^{n}\left(\frac{C_{L, j+1}^{n}+C_{L, j-1}^{n}}{2 \Delta y}\right) \\
& =D\left(\frac{-C_{L, j}^{n}-C_{L-1, j}^{n}}{(\Delta x)^{2}}+\frac{C_{L, j+1}^{n}-2 C_{L, j}^{n}+C_{L, j-1}^{n}}{(\Delta y)^{2}}\right) \\
& \quad-R C_{L, j}^{n}-Q_{L, j}^{n} .
\end{aligned}
$$

For the lower boundary condition, we have $1 \leq i \leq L$ and $j=1$, letting $C_{i, 0}^{n}=C_{i, 1}^{n}$. Substitute the approximate unknown vector nodes $C_{i, 0}^{n}$ of the lower boundary into (20) as follows:

$$
\begin{aligned}
& \frac{C_{i, 1}^{n+1}-C_{i, 1}^{n}}{\Delta t}+u_{i, 1}^{n}\left(\frac{C_{i+1,1}^{n}+C_{i-1,1}^{n}}{2 \Delta x}\right) \\
& \quad+v_{i, j}^{n}\left(\frac{C_{i, 2}^{n}+C_{i, 0}^{n}}{2 \Delta y}\right) \\
& =D\left(\frac{C_{i+1,1}^{n}-2 C_{i, 1}^{n}+C_{i-1,1}^{n}}{(\Delta x)^{2}}+\frac{C_{i, 2}^{n}-C_{i, 1}^{n}}{(\Delta y)^{2}}\right) \\
& \quad-R C_{i, 1}^{n}-Q_{i, 1}^{n} .
\end{aligned}
$$

For the upper boundary condition, we have $1 \leq i \leq L$ and $j=M$, letting $C_{i, M+1}^{n}=C_{i, M}^{n}$. Substitute the approximate unknown vector nodes $C_{i, M+1}^{n}$ of the upper boundary into (20) as follows:

$$
\begin{aligned}
& \frac{C_{i, M}^{n+1}-C_{i, M}^{n}}{\Delta t}+u_{i, M}^{n}\left(\frac{C_{i+1, M}^{n}+C_{i-1, M}^{n}}{2 \Delta x}\right) \\
& +v_{i, j}^{n}\left(\frac{C_{i, M+1}^{n}+C_{i, M-1}^{n}}{2 \Delta y}\right) \\
& =D\left(\frac{C_{i+1, M}^{n}-2 C_{i, M}^{n}+C_{i-1, M}^{n}}{(\Delta x)^{2}}+\frac{-C_{i, M}^{n}+C_{i, M-1}^{n}}{(\Delta y)^{2}}\right) \\
& \quad-R C_{i, M}^{n}-Q_{i, M}^{n} .
\end{aligned}
$$

\section{Numerical Simulations}

Assume that the western gate is opened and the water elevation along the gate is described as a function $f=1+$ $(0.25 \sin (0.1 t))$. The elevation of water on the eastern exit gates is assumed to be $\partial h / \partial x=\kappa=0.05$, where $\kappa$ is the rate of change between inner and outer water level through the eastern exit gate. The initial pollutant concentration in reservoir is $c_{0}=1.50\left(\mathrm{~kg} / \mathrm{m}^{3}\right)$. The water pollutant is released from the open western gate into the reservoir, which is the averaged pollutant concentration along the entrance gate
TABLE 1: Removal mechanism locations $(x, y)$ (meter, meter).

\begin{tabular}{lccc}
\hline Removal mechanism & Northern & Center & Southern \\
\hline Zone 1 & $(200,1600)$ & $(200,1080)$ & $(200,400)$ \\
Zone 2 & $(800,1600)$ & $(800,1080)$ & $(800,400)$ \\
Zone 3 & $(1200,1600)$ & $(1200,1080)$ & $(1200,400)$ \\
\hline
\end{tabular}

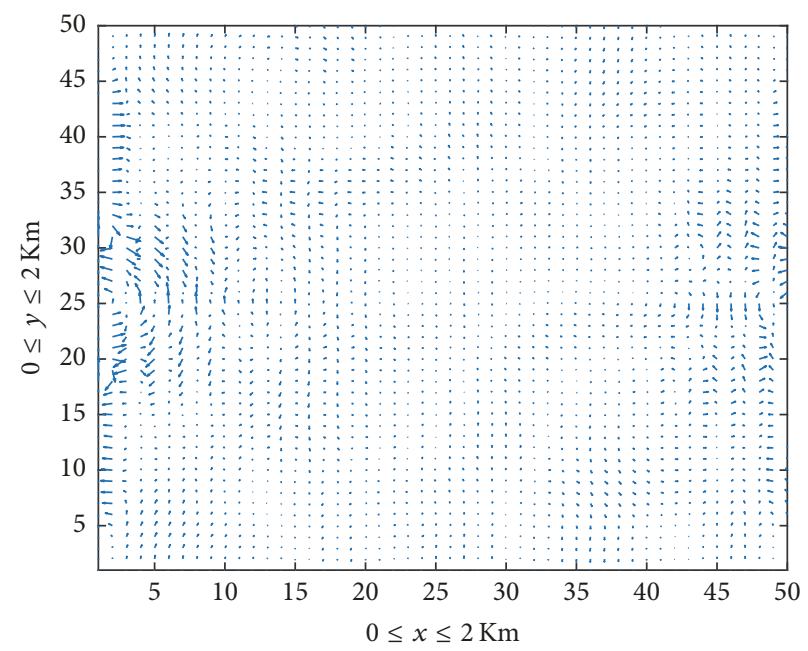

FIGURE 5: Approximated water velocity in an opened-closed reservoir.

defined by $c_{1}=10.00\left(\mathrm{~kg} / \mathrm{m}^{3}\right)$. The reservoir has drained water off through the exit eastern gate with the rate of change of pollutant concentration across the gate defined by $c_{2}=$ -0.001 . The embankment of reservoir is a nonabsorbing boundary bank. Consequently, there is no rate of change of pollutant concentration at the boundary of opened-closed reservoir, $\partial C / \partial n=0$, where $n$ is a normal vector. There are three contaminant removal mechanisms in the reservoir as shown in Figure 1. There are 3 mechanisms such as northern, center, and southern mechanisms which are contained in each zone. The removal mechanisms for each zone are located as shown in Table 1. If the contaminant removal mechanism is added, assume that the removal mechanisms have the same absorptive rate that can be described as a function $Q(x, y, t)=$ $0.10\left(\mathrm{~kg} / \mathrm{m}^{3} \mathrm{~s}\right)$. There is a pollutant concentration which is loss from the water surface, the decaying rate $R=0.1 \times 10^{-6}\left(\mathrm{~s}^{-1}\right)$.

The water flow velocity in $x$ - and $y$-directions and the water elevations are approximated by using (13) as shown in Tables 3 and 4 and Figure 5. The water pollutant concentrations are approximated by using (20). The approximated pollutant concentrations in an opened-closed reservoir are shown in Table 5.

4.1. Simulation 1: The Different Activated Zonal Removal Mechanism Systems Effect. The approximated pollutant concentration levels in removal mechanism system and nonremoval mechanism system are compared in Figure 6. The approximated pollutant concentrations, when the first zonal removal mechanism systems are activated, are shown as a surface plot in Figure 7. The approximated pollutant 
TABLE 2: Monitoring point locations $(x, y)$ (meter, meter).

\begin{tabular}{lccccc}
\hline Monitoring points & M1 & M2 & M3 & M4 & M5 \\
\hline Locations & $(1600,1600)$ & $(1600,1200)$ & $(1600,800)$ & $(1600,400)$ & $(2000,1000)$ \\
\hline
\end{tabular}

TABLE 3: Approximated water flow velocity in $x$-direction $u(x, y, t)(\mathrm{m} / \mathrm{s})$.

\begin{tabular}{lcccccc}
\hline$y / x(\mathrm{~m})$ & 0 & 400 & 800 & 1200 & 1600 & 2000 \\
\hline 800 & 0.0000 & 0.0870 & -0.0356 & 0.0411 & 0.0219 & -0.1939 \\
1000 & 0.0000 & -0.0344 & -0.0021 & 0.0103 & -0.0487 & 0.0305 \\
1200 & 0.0000 & -0.1260 & 0.0491 & -0.0584 & 0.0283 & 0.1866 \\
\hline
\end{tabular}

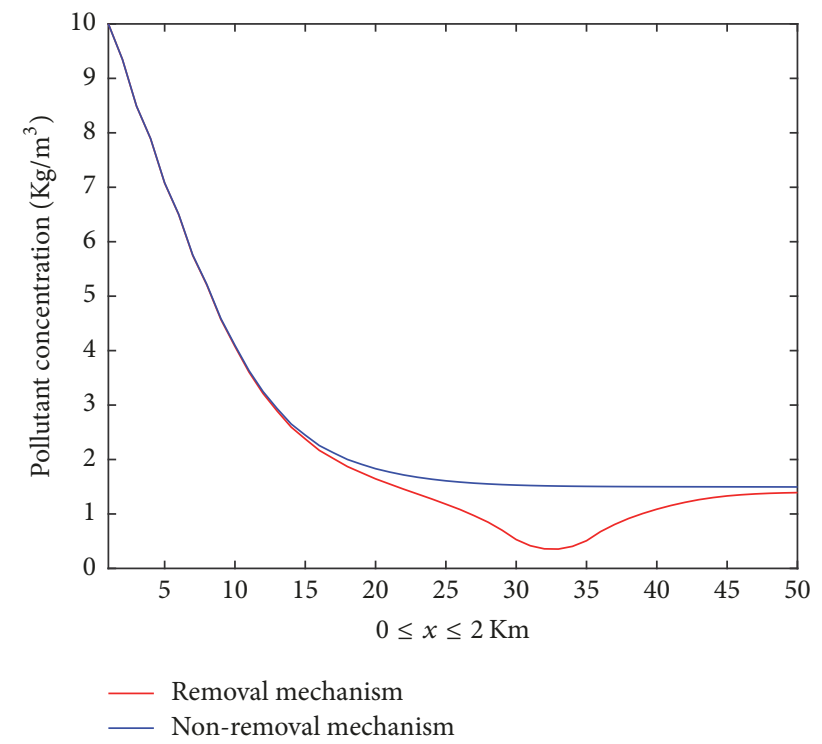

FIGURE 6: Comparison of approximated pollutant concentrations in removal mechanism system and nonremoval mechanism system.

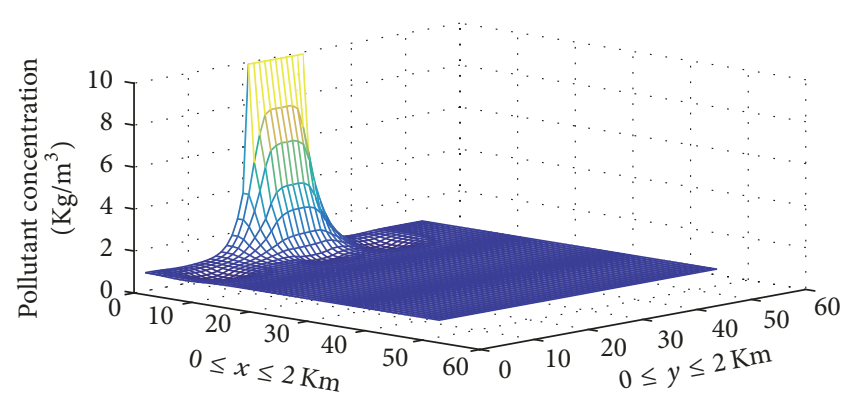

FIGURE 7: Approximated pollutant concentrations when the first zonal removal mechanism system is activated.

concentrations, when the second zonal removal mechanism systems are activated, are shown as a surface plot in Figure 8. The approximated pollutant concentrations, when the third zonal removal mechanism systems are activated, are shown as a surface plot in Figure 9.

The approximated pollutant concentrations along the northern part, $C(x, y, t)$, for all $0 \leq x \leq 2000 \mathrm{~m}, y=$ $1200 \mathrm{~m}$, and $0 \leq t \leq 100 \mathrm{~min}$, when the first, second, and third zonal removal mechanism systems are activated,

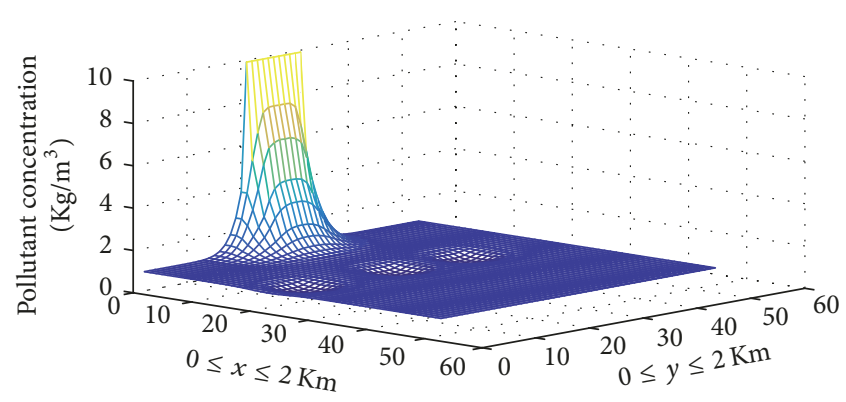

FIGURE 8: Approximated pollutant concentrations when the second zonal removal mechanism system is activated.

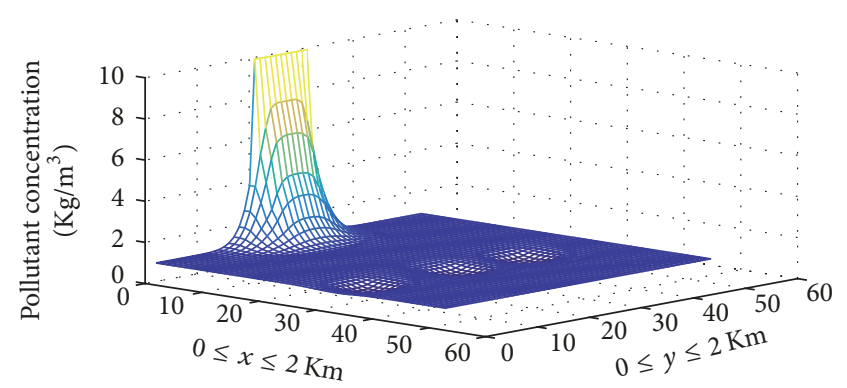

FIgURE 9: Approximated pollutant concentrations when the third zonal removal mechanism system is activated.

are compared in Figure 10. The approximated pollutant concentrations along the middle part, $C(x, y, t)$, for all $0 \leq$ $x \leq 2000 \mathrm{~m}, y=1000 \mathrm{~m}$, and $0 \leq t \leq 100 \mathrm{~min}$, when the first, second, and third zonal removal mechanism systems are activated, are compared in Figure 11. The approximated pollutant concentrations along the southern part, $C(x, y, t)$, for all $0 \leq x \leq 2000 \mathrm{~m}, y=800 \mathrm{~m}$, and $0 \leq t \leq 100 \mathrm{~min}$, when the first, second, and third zonal removal mechanism systems are activated, are compared in Figure 12.

4.2. Simulation 2: The Exit Gate Opening on Different Periods of Time Effect. Assuming that the third zonal removal mechanism systems are activated. The approximated pollutant concentrations over 5 monitoring nodes, when the third zonal removal mechanism systems are activated and the eastern exit gate is opened, are shown in Figure 13. Five monitoring nodes are located as shown in Table 2. If the eastern exit gate is opened or shut on different periods of 
TABLE 4: Approximated water flow velocity in $y$-direction $v(x, y, t)(\mathrm{m} / \mathrm{s})$.

\begin{tabular}{lcccccc}
\hline$y / x(\mathrm{~m})$ & 0 & 400 & 800 & 1200 & 1600 & 2000 \\
\hline 800 & 0.6213 & 0.0256 & -0.1082 & 0.0412 & 0.0323 & 0.1251 \\
1000 & -0.2959 & 0.4853 & -0.1423 & -0.0040 & -0.1365 & -0.5382 \\
1200 & 0.5779 & -0.0048 & -0.1376 & 0.1127 & 0.0168 & 0.1442 \\
\hline
\end{tabular}

TABLE 5: Approximated pollutant concentration $C(x, y, t)\left(\mathrm{kg} / \mathrm{m}^{3}\right)$.

\begin{tabular}{lcccccc}
\hline$y / x(\mathrm{~m})$ & 0 & 400 & 800 & 1200 & 1600 & 2000 \\
\hline 800 & 5.7854 & 2.9295 & 1.5580 & 0.7936 & 1.0637 & 1.3736 \\
1000 & 10.0000 & 4.0758 & 1.6448 & 0.5297 & 1.0873 & 1.3920 \\
1200 & 5.8178 & 2.9421 & 1.5798 & 0.9594 & 1.1355 \\
\hline
\end{tabular}

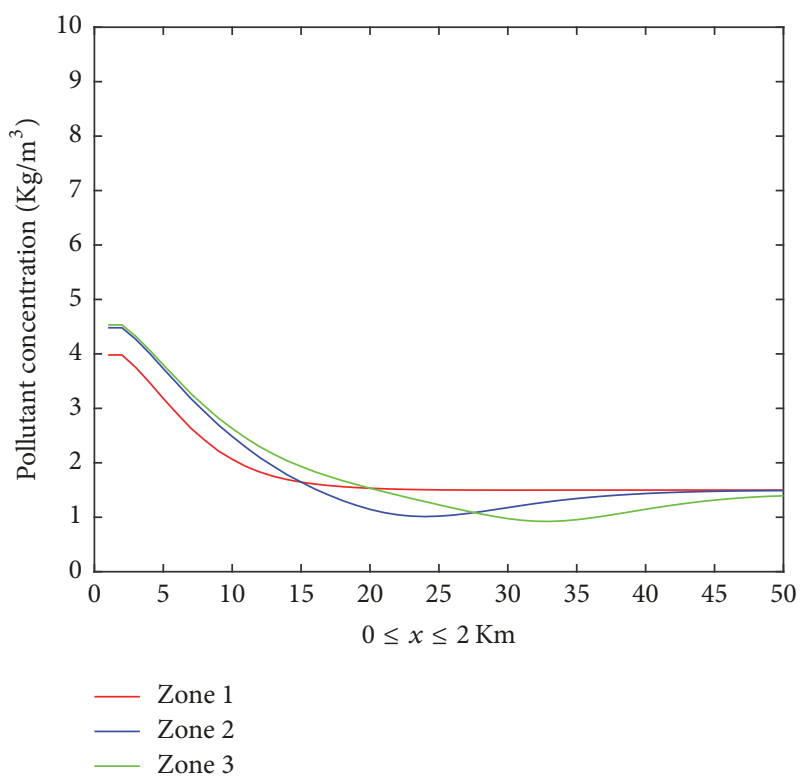

FIGURE 10: Comparison of approximated pollutant concentrations along the northern part, $C(x, y, t)$, for all $0 \leq x \leq 2000 \mathrm{~m}$ and $y=$ $1200 \mathrm{~m}$ and $0 \leq t \leq 100 \mathrm{~min}$, when the first, second, and third zonal removal mechanism systems are activated.

time such as $0-100 \mathrm{~min}, 33-100 \mathrm{~min}$, and $66-100 \mathrm{~min}$, the approximated pollutant concentrations over 5 monitoring nodes are also compared in Figure 13. The comparison of approximated pollutant concentrations along the eastern exit gate, $C(x, y, t)$, for all $800 \leq y \leq 1200 \mathrm{~m}, x=$ $2000 \mathrm{~m}$, and $0 \leq t \leq 100 \mathrm{~min}$, when the eastern exit gate is opened or shut on the difference periods of time such as $0-100 \mathrm{~min}, 33-100 \mathrm{~min}$, and $66-100 \mathrm{~min}$, is shown in Figure 14. The overall approximated pollutant concentrations, when the eastern exit gate is opened on the period 0-100 min, are shown in Figure 15. The overall approximated pollutant concentrations, when the eastern exit gate is opened on the period 33-100 min, are shown in Figure 16. The overall approximated pollutant concentrations, when the eastern exit gate is opened on the period $66-100 \mathrm{~min}$, are also shown in Figure 17.

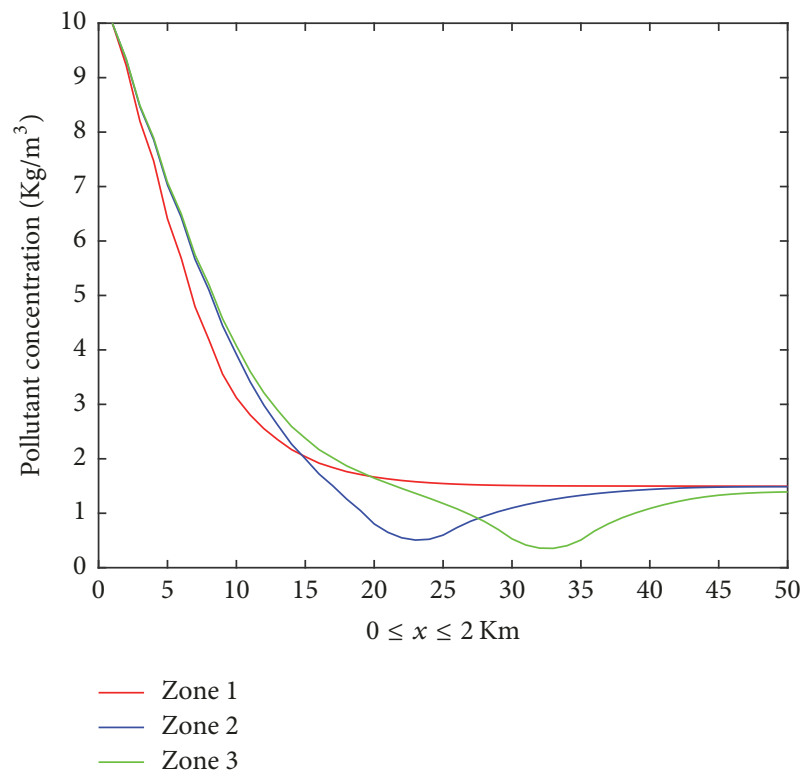

FIGURE 11: Comparison of approximated pollutant concentrations along the middle part, $C(x, y, t)$, for all $0 \leq x \leq 2000 \mathrm{~m}, y=1000 \mathrm{~m}$, and $0 \leq t \leq 100 \mathrm{~min}$, when the first, second, and third zonal removal mechanism systems are activated.

\section{Discussion}

In simulation 1, the different activated zonal removal mechanism systems are considered. The approximated pollutant concentration levels with the contaminant removal mechanism system are shown in Figure 6. We can see that the removal mechanism can be used to reduce the pollutant concentration in the opened-closed reservoir. The approximated pollutant concentrations, when the first/second/third zonal removal mechanism systems are activated, are shown as a surface plot in Figures 7, 8, and 9. The approximated pollutant concentrations along the northern/middle/southern parts, when the first, second, and third zonal removal mechanism systems are activated, are compared in Figures 10, 11, and 12. We can see that the third zonal removal mechanism systems give better water quality than another activated zones.

In simulation 2, the eastern exit gate opening on different periods of time is considered. The suitable third zonal 


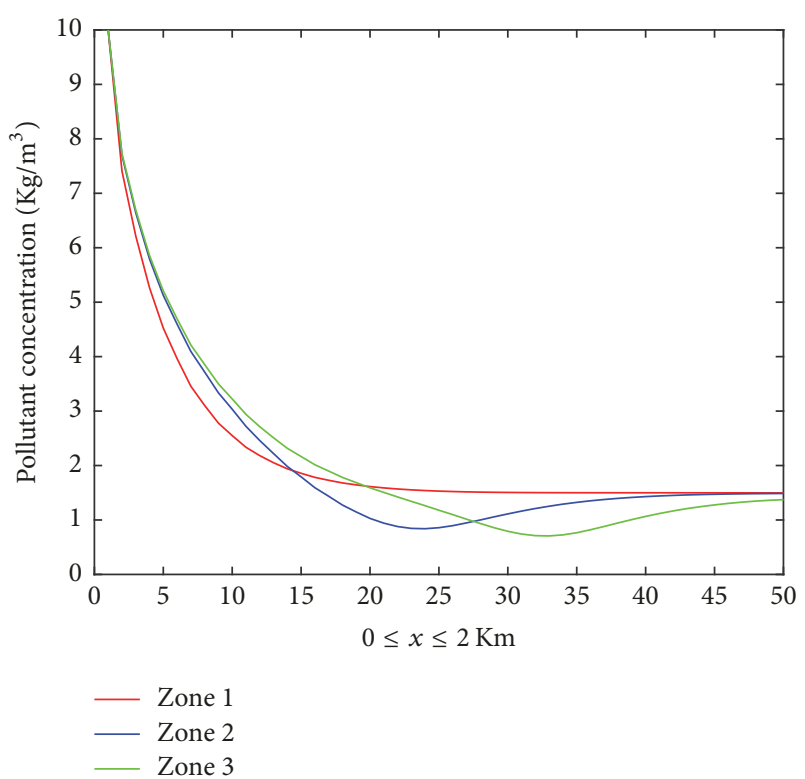

FIGURE 12: Comparison of approximated pollutant concentrations along the southern part, $C(x, y, t)$, for all $0 \leq x \leq 2000 \mathrm{~m}, y=$ $800 \mathrm{~m}$, and $0 \leq t \leq 100 \mathrm{~min}$, when the first, second, and third zonal removal mechanism systems are activated.

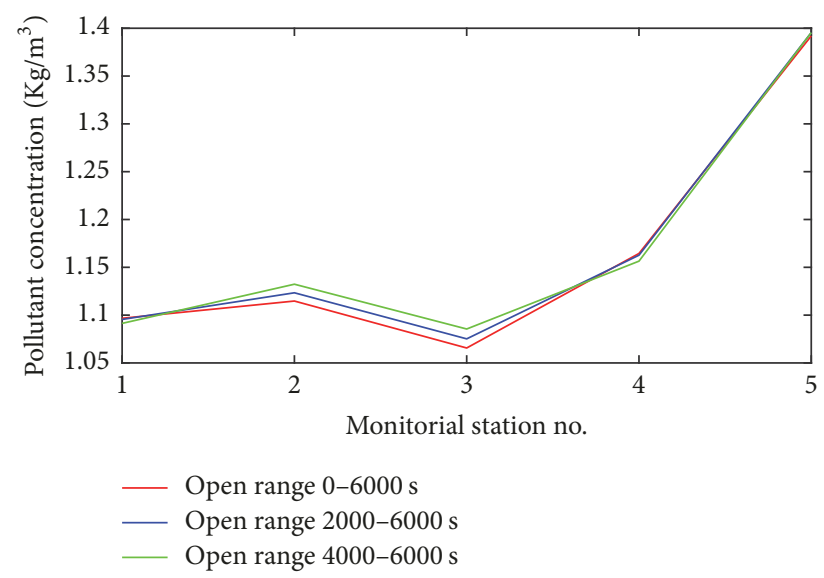

FIGURE 13: The approximated pollutant concentrations over 5 monitoring nodes, when the eastern exit gate is opened on $0-100 \mathrm{~min}$, 33-100 min, and 66-100 min.

removal mechanism system has been chosen to be activated. If the eastern exit gate is opened or shut on different periods of time such as $0-100 \mathrm{~min}, 33-100 \mathrm{~min}$, and $66-100 \mathrm{~min}$, the approximated pollutant concentrations over 5 monitoring nodes are shown in Figure 13. We can see that the full periods (0-100 $\mathrm{min})$ of opened exit gate give better water quality for all 5 monitoring nodes.

If we consider the approximated pollutant concentrations along the eastern exit gate, when the the eastern exit gate is opened or shut on the difference periods of time as shown in Figure 14, we can also see that the long opened exit gate period $(0-100 \mathrm{~min})$ gives the better monitored water quality than another periods as well.

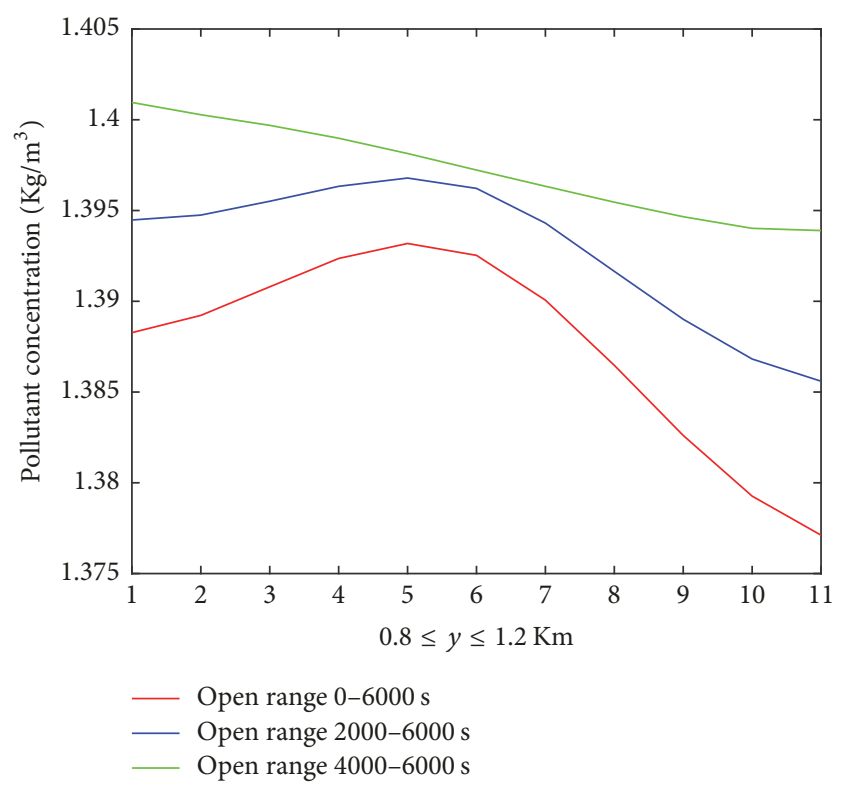

FIGURE 14: The approximated pollutant concentrations over the eastern exit gate is opened on 0-100 $\mathrm{min}, 33-100 \mathrm{~min}$, and 66-100 min, when $C(x, y, t)$ for all $800 \leq y \leq 1200 \mathrm{~m}$ and $x=2000 \mathrm{~m}$.

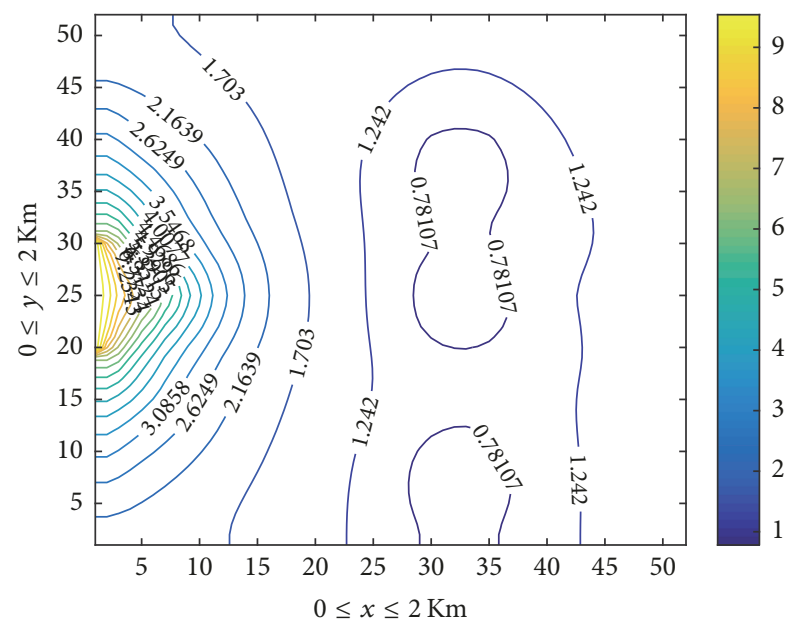

FIGURE 15: The overall approximated pollutant concentrations when the eastern exit gate is opened on the period 0-100 min.

However, the overall approximated pollutant concentrations, when the eastern exit gate is opened on the different period of times, are shown in Figures 15, 16, and 17. We can see that the period $(66-100 \mathrm{~min})$ of the opened exit gate, which is shortest, gives better overall water quality than other periods. These mean that the monitoring point placement for water quality measurement in an opened-closed reservoir affect to water quality assessment. These mean that the approximated water quality measurement on monitoring points in an opened-closed reservoir affect the accuracy assessment. The overall simulated pollutant concentration method gives more precise water quality assessment than the monitoring assessment method. 


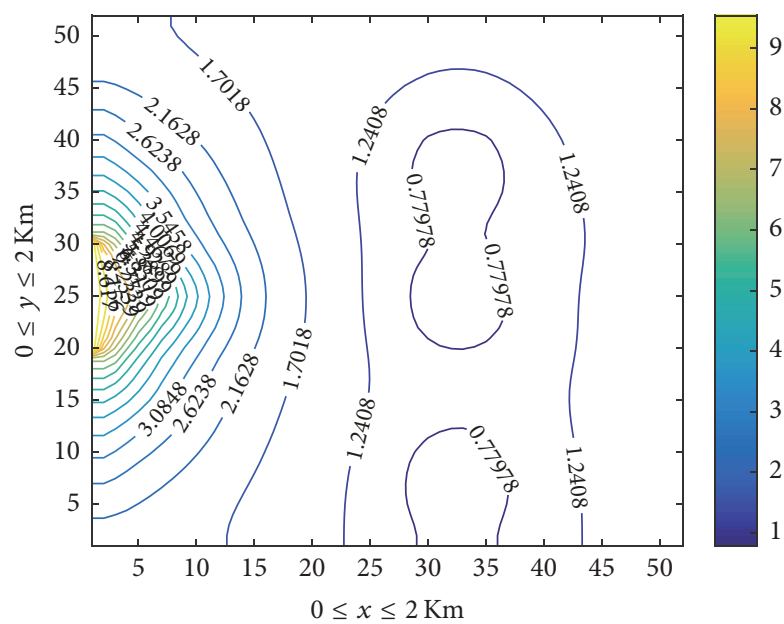

FIGURE 16: The overall approximated pollutant concentrations when the eastern exit gate is opened on the period 33-100 min.

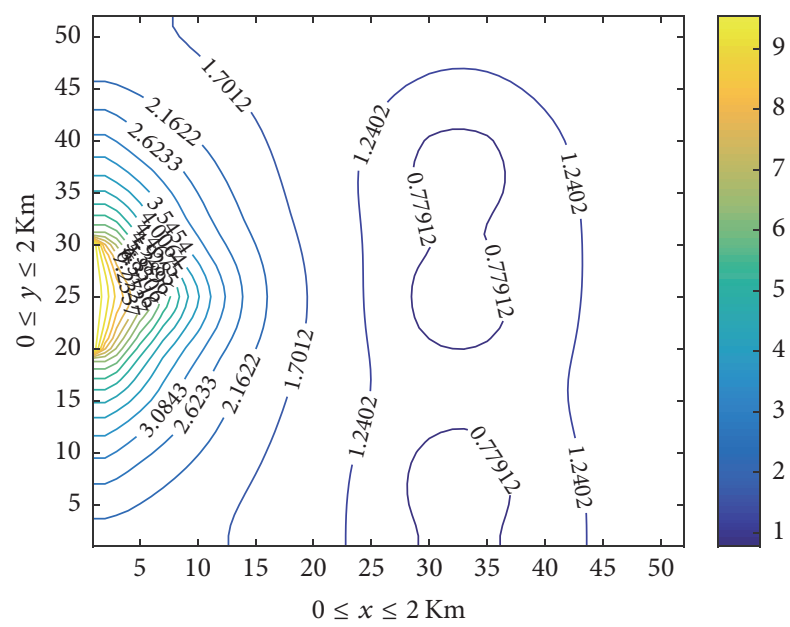

FIGURE 17: The overall approximated pollutant concentrations when the eastern exit gate is opened on the period $66-100 \mathrm{~min}$.

\section{Conclusion}

The monitoring point placement for water quality measurement in an opened-closed reservoir can give accurate or inaccurate assessment. However, the proposed numerical simulations have given the overall approximated water quality when the removal mechanism systems are activated in different zones. The proposed techniques give the zonal removal mechanism placement to specialists who want to control the water quality. Moreover, we obtain the overall and specified approximated water quality for each points and time when the exit gate is opened on the different periods of time. The techniques can give a suitable period of time to open the exit gate to specialists who want to get a good agreement water quality by using contaminant removal mechanism.

\section{Conflicts of Interest}

The authors declare no conflicts of interest.

\section{Acknowledgments}

This research is supported by the Centre of Excellence in Mathematics, the Commission on Higher Education, Thailand.

\section{References}

[1] P. Tabuenca, J. Vila, J. Cardona, and A. Samartin, "Finite element simulation of dispersion in the Bay of Santander," Advances in Engineering Software, vol. 28, no. 5, pp. 313-332, 1997.

[2] P. Tabuenca, J. Cardona, and A. Samartín, "Numerical model for the study of hydrodynamics on bays and estuaries," Applied Mathematical Modelling, vol. 16, no. 2, pp. 78-85, 1992.

[3] N. Pochai, S. Tangmanee, L. J. Crane, and J. J. H. Miller, "A mathematical model of water pollution control using the finite element method," Proceedings in Applied Mathematics and Mechanics, vol. 6, no. 1, pp. 755-756, 2006.

[4] N. Pochai, S. Tangmanee, L. J. Crane, and J. J. H. Miller, "A water quality computation in the uniform reservoir," Journal of Interdisciplinary Mathematics, vol. 12, no. 1, pp. 19-28, 2009.

[5] A. Garzon and L. D’ALpaos, "Modified method of characteristics technique combined with Galerkin finite element method to solve shallow water mass transport problems," in Proceedings of the 23rd International Conference on Coastal Engineering, pp. 3068-3080, October 1992.

[6] N. Pochai, "A numerical computation of a non-dimensional form of stream water quality model with hydrodynamic advection-dispersion-reaction equations," Nonlinear Analysis: Hybrid Systems, vol. 3, no. 4, pp. 666-673, 2009.

[7] N. Pochai, "A numerical treatment of nondimensional form of water quality model in a nonuniform flow stream using Saulyev scheme," Mathematical Problems in Engineering, Article ID 491317, 2011.

[8] N. Pochai, "Unconditional stable numerical techniques for a water-quality model in a non-uniform flow stream," Advances in Difference Equations, Paper No. 286, 13 pages, 2017.

[9] N. Pochai, "A Computation of a Non-linear Hydrodynamic Model in a Uniform Reservoir," in 5th Asian Mathematical Conference Proceedings, vol. II, pp. 596-600, June 2009.

[10] N. Pochai and C. Sornsri, "A non-dimensional form of hydrodynamic model with variable coefficients in a uniform reservoir using Lax-Wendroff method," in Proceedings of the 2nd International Science, Social Science, Engineering and Energy Conference 2010, I-SEEC 2010, pp. 89-93, Thailand, December 2010.

[11] N. Pochai, S. Tangmanee, L. J. Crane, and J. J. H. Miller, "A water quality computation in the uniform channel," Journal of Interdisciplinary Mathematics, vol. 11, no. 6, pp. 803-814, 2008.

[12] W. Klaychang and N. Pochai, "A numerical treatment of a nondimensional form of a water quality model in the Rama-nine reservoir," Journal of Interdisciplinary Mathematics, vol. 18, no. 4, pp. 375-394, 2015.

[13] C. R. Robinson, Shallow Water Equations Lecture Notes, PHY 307, Syracuse University, 2011.

[14] K. Thongtha and J. Kasemsuwan, "Analytical solution to a hydrodynamic model in an open uniform reservoir," Advances in Difference Equations, Paper No. 149, 9 pages, 2017.

[15] H. Ninomiya and K. Onishi, "Flow Analysis Using a Personal Computer," Computational Mechanics Publications, 1991. 
[16] C. B. Vreugdenhil, "Three-dimensional shallow-water flow," in Numerical Methods for Shallow-Water Flow, vol. 13 of Water Science and Technology Library, pp. 217-246, Springer Netherlands, Dordrecht, 1994. 


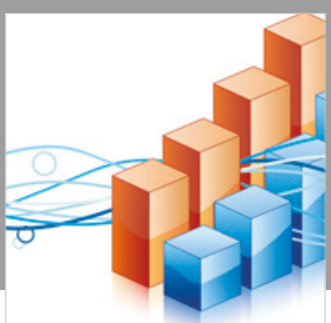

Advances in

Operations Research

\section{-n-m}
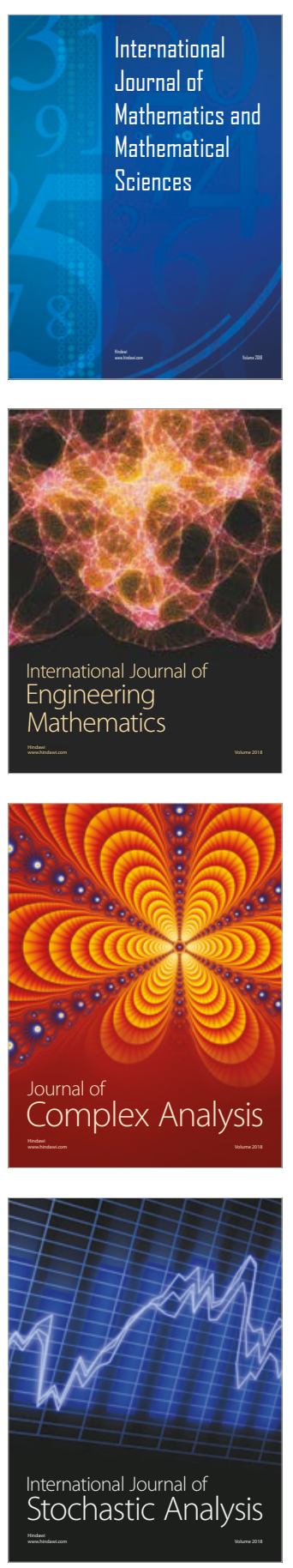
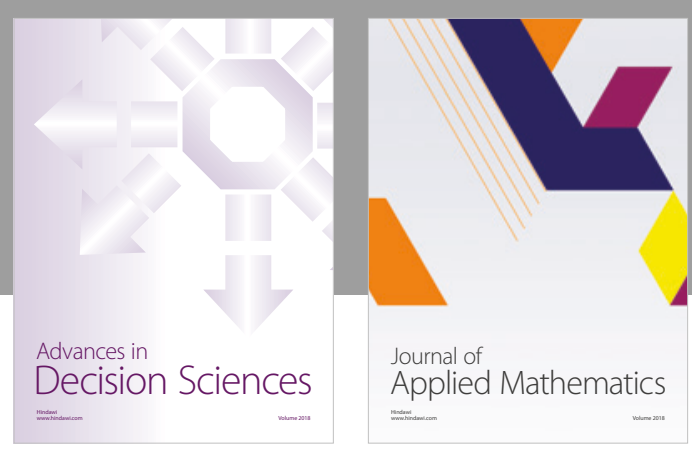

Journal of

Applied Mathematics
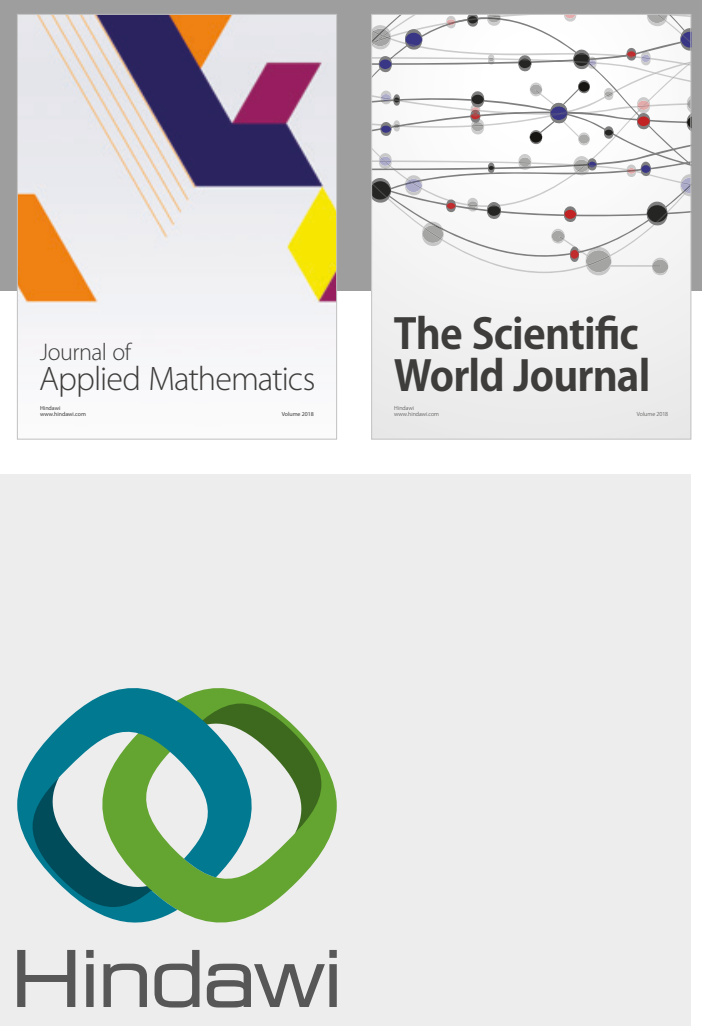

Submit your manuscripts at

www.hindawi.com

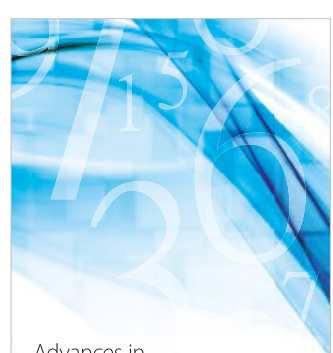

Advances in
Numerical Analysis
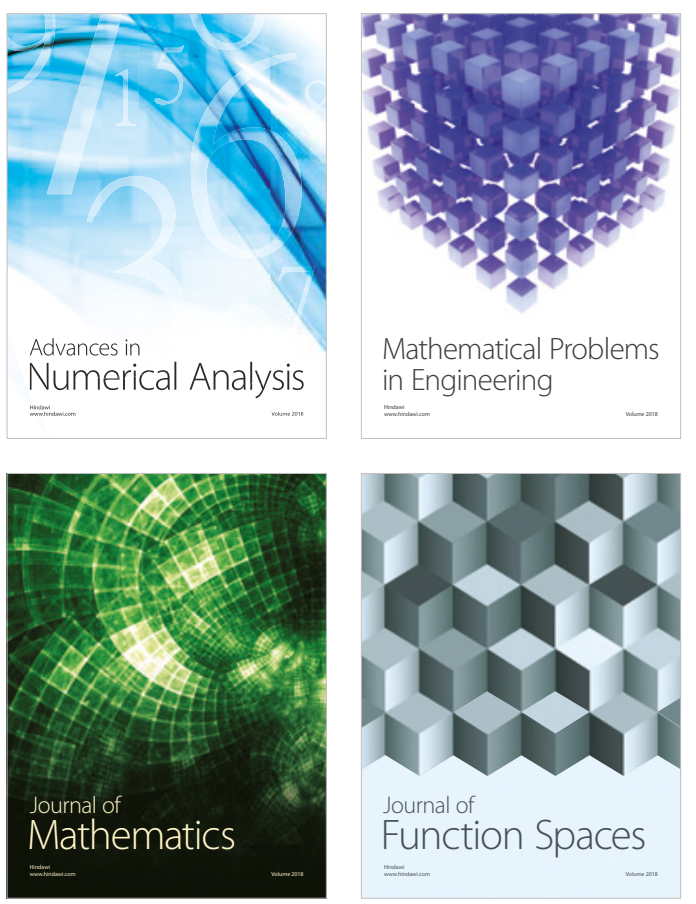

Mathematical Problems in Engineering

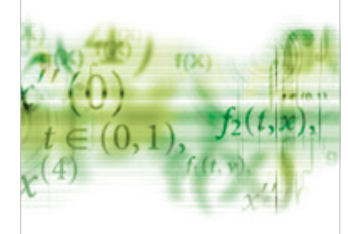

International Journal of

Differential Equations

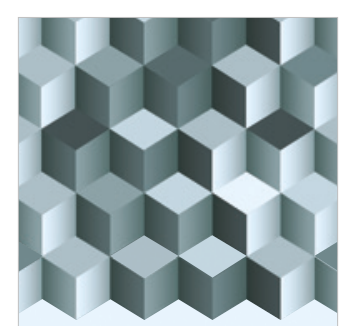

Journal of

Function Spaces

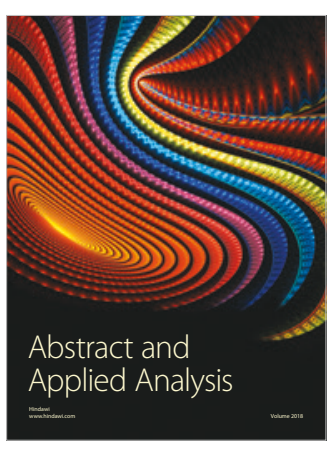

The Scientific

World Journal

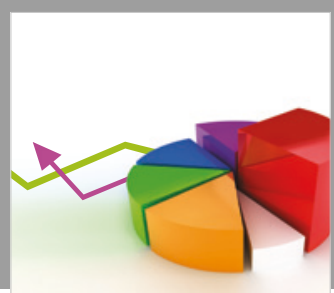

Journal of

Probability and Statistics
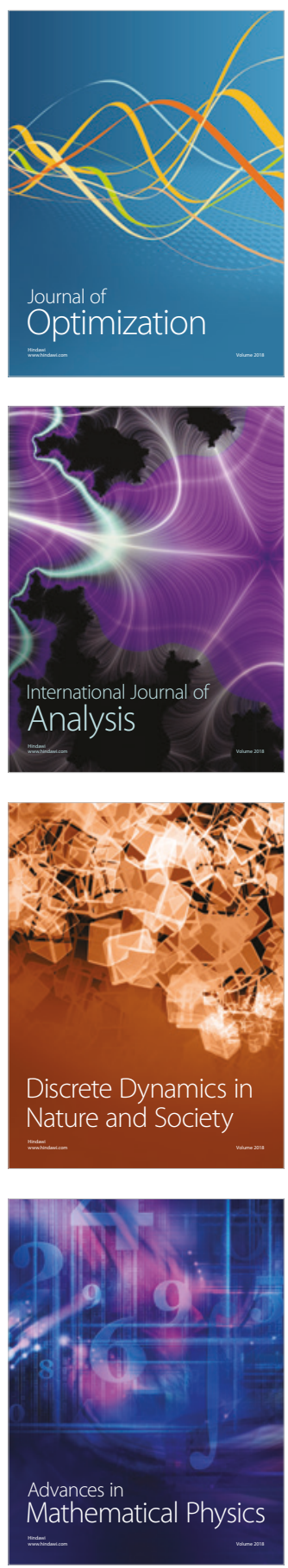